Erratum

\title{
On the Low Energy Limit of Reflection and Sticking Coefficients in Atom Surface Scattering
}

\section{Long Range Forces}

J. Böheim and W. Brenig

Physik-Department Technische Universität München, Garching,

Federal Republic of Germany

J. Stutzki

I. Physikalisches Institut der Universität, Köln, Federal Republic of Germany

Z. Phys. B - Condensed Matter 48, 43-49 (1982)

The following corrections on the galley proofs were, inadvertently, not taken into account:

page 45, 1st line after Eq. (2.24) should read:

"Notice that here as in (2.17) the $\tan (2 g-3 \pi / 4)$ can..."

page 46 , Eq. (2.26), $/ \mathrm{kg}^{2}$ ) should read:

$/\left(\mathrm{kg}^{2}\right)$. "that" in the third line after (2.26) should be deleted.

page 46, Eq. (2.28):

The "." should be ommitted and the next line should continue with "in".

page 47, the present Fig. 5 should be added to the present Fig. 4. The new Fig. 5 should read:

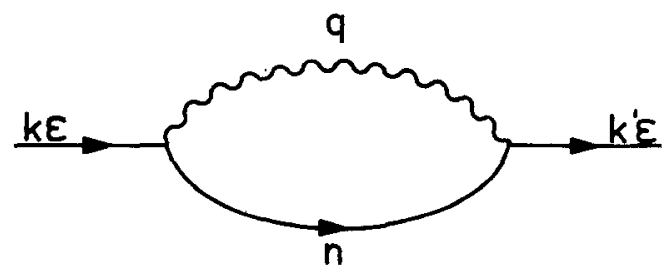

page 47,2 nd line from below, " $K$ " should read: " $k$ ".

page 48 , Eq. (3.12): “ $\chi\left(n, \varepsilon_{k}\right)$ " should be replaced by " $\gamma\left(n, \varepsilon_{k}\right)$ ".

17nth line from below: "... energy shift ..."

3rd line below Fig. 7 caption should read:

"these effects properly".

caption of Fig. 6, 3rd. line from $D$ on should read:

" $D=161 \mathrm{~K}, \Theta_{D}=380 \mathrm{~K}, \ldots$,
J. Böheim
W. Brenig
Physik-Department
Technische Universität München
Theoretische Physik
James-Franck-Strasse
D-8046 Garching bei München
Federal Republic of Germany
J. Stutzki
I. Physikalisches Institut
Universität zu Köln
Zülpicher Strasse 77
D-5000 Köln 41
Federal Republic of Germany 